\title{
Vascular Patterns of the Rat Hippocampal Formation
}

\author{
Peter Coyle ${ }^{1}$ \\ Department of Anatomy, The University of Michigan Medical School, \\ Ann Arbor, Michigan 48109
}

Reccived April 23, 1976

\begin{abstract}
Little is known of the course and distribution of blood vessels supplying and draining the hippozampus. Such information could be of value in designing and evaluating lesion and ablation experiments and may reflect spatial properties of neurons. This study mapped the distribution of major arteries and veins of the rat hippocampal formation. Arteries and veins of adult female Wistar rats anesthetized with sodium pentobarbital were injected with silicone rubber. Double injections to demonstrate both arteries and veins in the same animal were with India ink and a fluorescent material. Arterial supply to the hippocampus was via transverse hippocampal arteries that stemmed from the longitudinal hippocampal artery, a branch of the posterior cerebral artery. Internal transverse hippocampal arteries located in the hippocampal fissure supplied small, short branches to the adjacent blade of the fascia dentata, part of the area dentata, and CA3 fields. Other branches of the longitudinal artery supplied the remaining blade of the fascia and area dentata, subicular fields, and entorhinal structures. Internal transverse hippocampal veins located in the hippocampal fissure alternated in position with the arteries and appeared to be paired with, and to drain fields supplied by, the internal transverse arteries. Deep transverse hippocampal veins, unaccompanied by arteries, received branches in the intraventricular alveus and adjacent stratum oriens of CA3. The transverse veins drained into longitudinal vessels or the basal vein. Although transversely directed arteries and veins may suggest a hippocampal lamellar neuronal organization, microvascular fields must be mapped before claims are made for a totally segmental vascular architecture in the hipposampus.
\end{abstract}

\section{INTRODUCTION}

Published information on identified hippocampal formation ${ }^{2}$ blood vessels and the structures supplied and drained in experimental animals

1 The author expresses appreciation to Mr. W. L. Brudon for preparation of the illustrations and to Ms. Camilla Broman for typing the manuscript and to the University of Michigan Medical School for publication support.

2 The term "hippocampal formation" was used to include the various fields of the hippocampus (or cornu Ammonis (CA) segments CA1-4 as designated by Lorente 
is scant. Nilges (12) investigated arteries to cornu Ammonis of dog, cat, rabbit, opossum, guinea pig, and rhesus monkey. In all species, he observed rakelike arteries arising from the posterior cerebral artery or its branches to enter the hippocampal fissure in route to supply the cornu Ammonis proper. In rabbit, Andersen et al. (2) noted similarly arranged arteries and suggested some veins may have corresponding spatial arrangements. Preliminary studies by Coyle $(8,9)$ noted arteries within the hippocampal fissure. Hippocampal veins unaccompanied by arteries were associated with the intraventricular alveus (7).

The rakelike appearance of arteries in the hippocampal fissure might relate to a similar organization of veins. Furthermore, such a venous pattern could suggest the pairing of arteries and veins. Findings of paired arteries and veins could lend more support to the theory that the hippocampus has a lamellar or segmental organization. The present investigation was undertaken to determine which arteries and veins were possibly paired and to map their spatial positions to gain better impressions of vascular and neural relations in this brain region.

\section{METHODS AND MATERIALS}

Female Wistar rats weighing between 180 and $275 \mathrm{~g}$ were anesthetized with sodium pentobarbital ( $50 \mathrm{mg} / \mathrm{kg}$ body weight, ip). Arterial injections were via the left ventricle with a number 15 cannula inserted into the ascending aorta and secured with a ligature placed proximal to the brachiocephalic artery. The descending aorta distal to the left common carotid artery was clamped. Venous injections were into the external jugular vein. Some brains were perfused with physiologic saline prior to injection of other media.

Three types of display media were utilized. Arteries in 12 brains were investigated following arterial injections with General Electric RTV Silicone Rubber diluted with RTV 910 Diluent. Veins of 10 brains were studied following injection of 5 to $10 \mathrm{ml}$ Dow Corning $3111 \mathrm{RTV}$ En-

de Nó $(10,11))$, the fascia dentata, and subicular and entorhinal fields. Attempts to distinguish various subfields of $\mathrm{CA} 3$ were not warranted by virtue of the type of preparations. The pyramidal and granule cell layers were evident in cleared sections allowing for identification of strata superficial and deep to the cell layers. The two blades of the fascia dentata were named in reference to their position to the hilum formed by CA1-3. The fascia dentata external blade located external to the hilum was on the side of the extraventricular alveus. The internal blade bordered the hippocampal fissure. The longitudinal hippocampal axis extended toward the septum in one direction and toward the temporal region in the other. The transverse hipposampal axis was approximately normal to the longitudinal one. Such terminology was used to avoid the necessity for translation at different septal-temporal locations and yet maintain correct orientation of reference points. 
capsulant diluted with number 200 dielectric fluid. India ink was used to demonstrate both arteries and veins in eight brains. The best specimens were obtained when undiluted Higgins or Winsor Newton India ink was introduced into the beating ventricle and 10 to $20 \mathrm{ml}$ was administered following cardiac arrest. Ten animals were double injected, that is, India ink was injected followed by 5 to $10 \mathrm{ml}$ Craftint daylight water-base fluorescent paint into either the arteries or the veins. Paint particle sizes were such that the material did not pass into the capillary bed. Perfusion pressures were not monitored and pressure was applied by hand to the syringe plunger.

All brains were fixed following immersion in $10 \%$ neutral buffered formalin for at least 3 days. Most brains injected with the rubber compounds were dissected. India ink and double-injected specimens were dissected or sectioned with a hand-held razor blade. Sections less than $1 \mathrm{~mm}$ in thickness were cleared and mounted in glycerin on glass slides. The coverslip was sealed with Permount. Observations were with light and dissecting microscopes and sketches of vessels were made. Sections of specimens injected with the fluorescent material were observed with a xenon light source. The excitor filter was a Kodak number 47 and number 15 and 22 gelatin barrier filters were used. The investigative strategy was to first map gross vessels in dissected specimens, then confirm and study smaller vessels using the sectioned brains.

\section{ARTERIAL SUPPLY TO THE HIPPOCAMPAL FORMATION}

The Longitudinal Arteries. In rat, the major vascular supply to the hippocampal formation was via branches of the basilar and internal carotid arteries. The posterior communicating artery, a branch of the internal carotid artery, joined a usually smaller diameter posterior cerebral artery in the region of the basis peduncle. The posterior cerebral artery coursed over the peduncle and ascended in a rostrodorsal direction over the midbrain and caudal diencephalon. In course several branches arose from the posterior cerebral vessel (Fig. 1).

The longitudinal hippocampal artery, a branch of the posterior cerebral artery, followed a path nearly parallel to the vessel of supply. Both vessels coursed approximately parallel to the longitudinal axis of the hippocampus and were in close proximity to the porta of the hippocampal fissure. The vessels frequently were of nearly equal diameter and spiraled around each other. Numerous segmental arteries, mostly from the longitudinal hippocampal artery, entered the obliterated hippocampal fissure and others extended over the extrahilar surface of the fascia dentata to course in the 


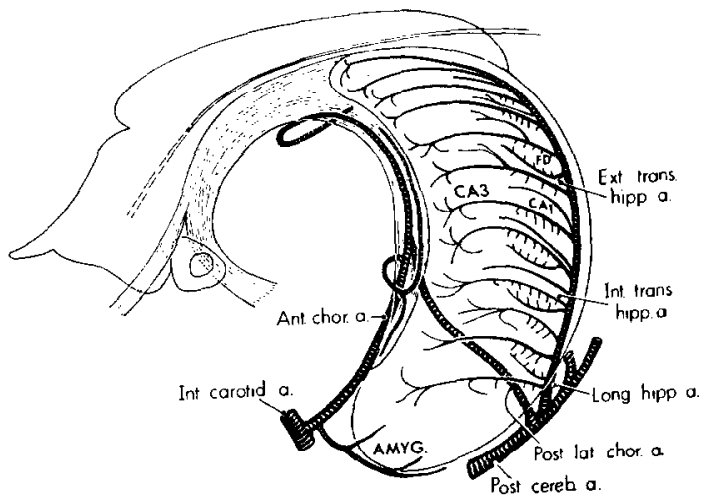

FIg. 1. Lateral view of the longitudinal hippocampal artery and branches supplying the CA3 fields and fascia dentata. Abbreviations in this and subsequent figures: Ainternal transverse hippocampal artery, AMYG-amygdala, Ant. chor. a.-anterior choroidal artery, Basal v.-basal vein, CA-cornu Ammonis, CA1-cornu Ammonis segment 1, CA3 - cornu Ammonis segment 3, CC-corpus callosum, Ext. long v.external longitudinal vein, Deep long v.-deep longitudinal vein, Deep trans. hipp. vs.-deep transverse hippocampal veins, Ext. long. v.--external longitudinal vein, Ext. trans. hipp. a.--external transverse hippocampal artery, Ext. trans. hipp. vs.-external transverse hippocampal veins, FD-fascia dentata, F. dent.-fascia dentata, Hipp.hippocampus, Hipp. fissure-hippocampal fissure, Int. carotid a.-internal carotid artery, Int. trans. hipp. a.-internal transverse hippocampal artery, Int. trans. hipp. vs.internal transverse hippocampal veins, Long. hipp. a.-longitudinal hippocampal artery, Long. hipp. v.--longitudinal hippocampal vein, Post. cereb. a.--posterior cerebral artery, Post. lat. chor. a.-posterior lateral choroidal artery, $\mathrm{R} P \mathrm{O}-$ stratum radiatum, stratum pyramidale, stratum oriens, $\mathrm{V}$-internal transverse hippocampal vein.

fimbriodentate fissure or extended parallel to the surface of the extraventricular alveus.

Distribution patterns of other branches of the posterior cerebral artery including the posterior lateral choroidal artery, the posterior medial choroidal artery, and the transverse collicular artery were described elsewhere (8) and are not included here for no branches were traced to hippocampal formation structures in the rat.

Formation of the Transverse Hippocampal Arteries. Several types of transverse hippocampal arteries were observed. The largest diameter, longest, and most consistently spaced vessels were the internal transverse hippocampal arteries. (Figs. 1,2). At the septal pole of the hippocampus the vessels followed an oblique course nearly parallel to the sagittal plane while angulation was less at the temporal pole and least at the midpolar region. At the midpolar region the vessels nearly paralleled the transverse hippocampal plane in their rostral projection. The internal transverse arteries usually arose from the longitudinal hippocampal artery and traveled 
in the obliterated hippocampal fissure to its depth where numerous branches were formed in the pit. The artery or its branches penetrated stratum lacunosum et moleculare, stratum radiatum and lucidum, and stratum pyramidale to supply those nonhilar structures of CA3. Small vessels extended into the intraventricular portion of CA3 stratum oriens. In course, small-diameter branches of the internal arteries penetrated the molecular layer of the fascia dentata internal blade and occasionally large branches extended through stratum granulosum. Branches of the internal transverse hippocampal arteries projected around the tip of the fascia dentata internal blade to supply, in part, cornu Ammonis segments in the hilum of the fascia denata.

The external transverse hippocampal arteries were shorter, of smaller diameter, did not branch as profusely, or supply as much tissue as compared to the internal vessels (Fig. 2). The external arteries were more variable in number and course, and arose from the internal transverse hippocampal arteries or the longitudinal hippocampal artery. The external arteries were more evident at midpolar and temporal hippocampal regions and supplied small-diameter vessels that penetrated the molecular layer of the fascia dentata external blade. The external vessels located in the fimbriodentate fissure supplied branches to hilar structures including the cornu Ammonis segments. At midpolar and temporal regions of the

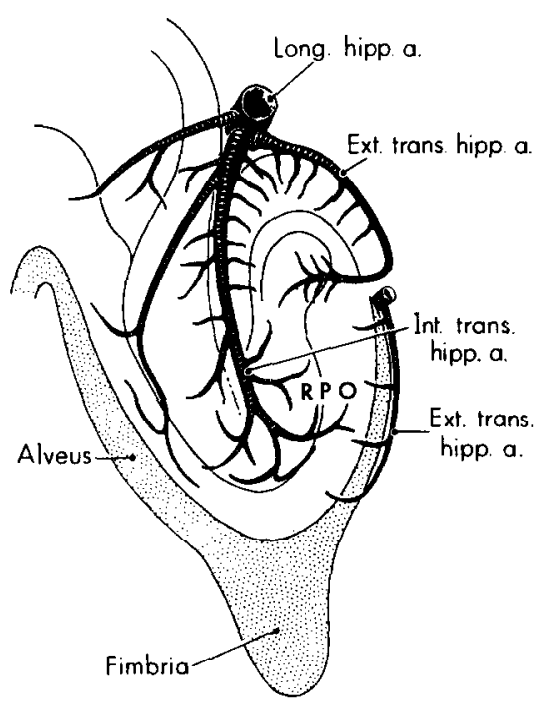

FIG. 2. Illustration of a horizontal section through the hippocampal formation at the midpolar region showing arteries supplying cornu Ammonis fields and the fascia dentata. 


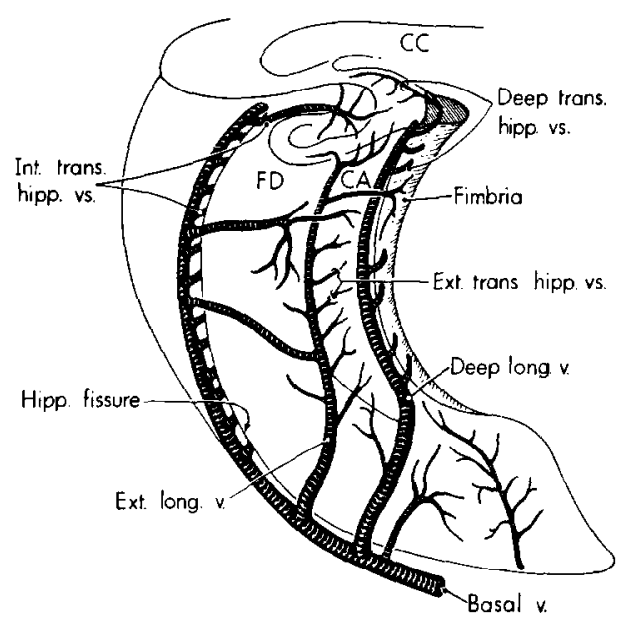

FIG. 3. Medial view of the hipposampal formation illustrating the longitudinal and transverse hippocampal veins draining the cornu Ammonis.

hippocampus the vessels followed a course in the transverse planc parallcl to the extraventricular alveus to supply it and the extraventricular CA3 stratum oriens.

Additional arteries stemmed from the longitudinal vessels or the internal transverse hippocampal arteries to supply subicular and possibly CA1 fields (Fig. 2). These vessels were shorter, of smaller diameter, and with fewer major branches compared to the internal transverse arteries supplying CA3 fields. Still other larger-diameter, longer arteries coursed obliquely from a ventromedial to dorsolateral position over the surface of the entorhinal cortex, supplying it with smaller branches before anastomosing with branches of the middle cerebral artery in neocortical regions.

The Longitudinal Veins. Several different veins were positioned nearly parallel or at an angle to the direction of the longitudinal hippocampal axis (Fig. 3). The largest-diameter, most consistently placed vessel paralleled the course of the longitudinal artery. The basal vein was positioned near the porta of the hippocampal fissure and received from numerous internal transverse hippocampal veins. Anastomoses occurred with the transverse sinus and the external and deep longitudinal veins. The basal vein joined the tentorial vein and others ventrally and anastomosed with veins draining into the transverse sinus dorsally. A smaller vein drained the temporal pole of the hippocampal formation and followed a course parallel to the fimbria before draining into the basal vein.

The Transverse Hippocampal Veins. The deep transverse hippocampal 


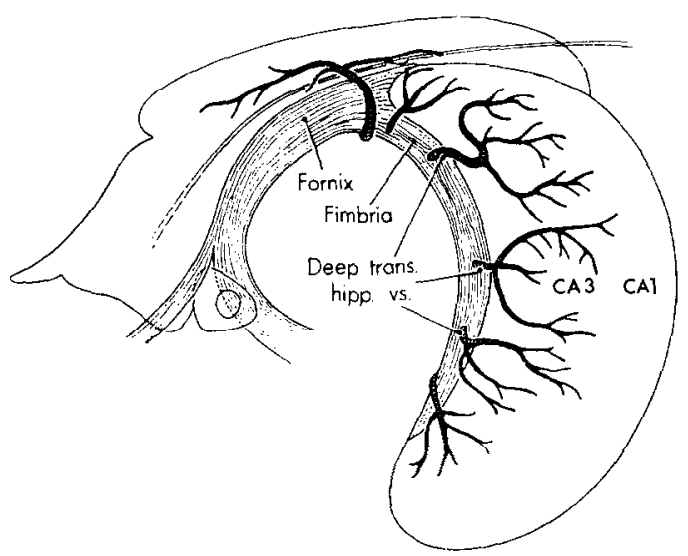

FIG. 4. Lateral view of the hippozampus illustrating deep transverse veins associated with the intraventricular alveus. Note the smaller vessels join to form chaliceshape networks. The stem vessels penetrate the fimbria in most cases.

veins were most readily observed in India ink preparations but were seen in specimens injected with silicone rubber where the overlying neocortex was dissected to expose the intraventricular alveus. Intraventricular CA3 fields nearest the fimbria were drained rostrally by small veins which often joined to form chalice shaped networks (Fig. 4). The vessels were located in the alveus or at the alvear-stratum oriens interface. Branches

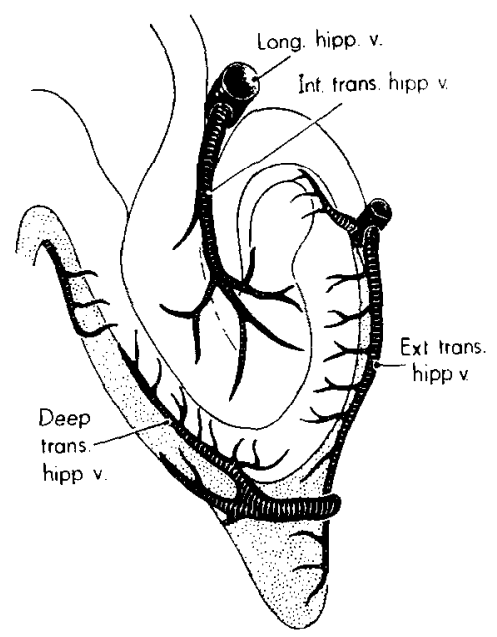

FIG. 5. Illustration of a horizontal section through the hippocampal formation at the midpolar region showing veins draining the cornu Ammonis and fimbria. 


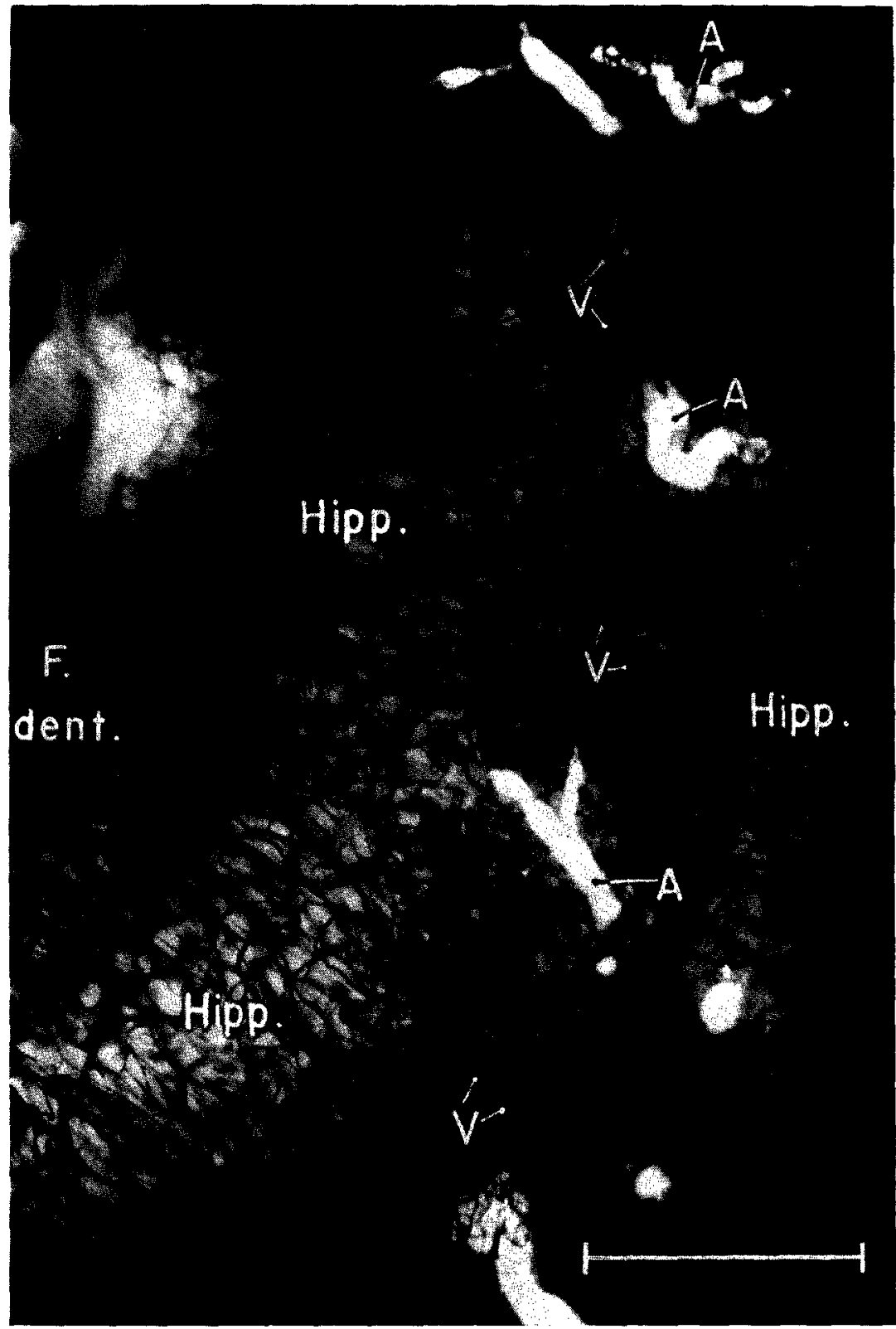

FIG. 6. Coronal section through the hippocampus and fascia dentata near the midpolar region. Note the alternation of the internal transverse hippocampal arteries (A) and internal transverse hipposampal veins $(V)$ in the hippocampal fissure. Scale 0.4 $\mathrm{mm}$. See Fig. 1 for abbreviations. 
were traced into stratum oriens. The stem or trunk vessel of the chalice formed by convergence of several small transversely directed veins penetrated the fimbria, usually near its junction with the alveus, to occupy a position in the transverse cerebral fissure. Three to five trunks were ordinarily observed to penetrate the fimbria along its longitudinal extent. The trunks were more commonly related to the septal and midpolar regions of the hippocampus but were occasionally observed near the temporal pole as well. The small veins were not traced the entire transverse length of the intraventricular alveus in glycerin-cleared specimens. At least part of CA1 and all the subicular fields were associated with vessels draining in a caudal direction (not illustrated).

The internal transverse hippocampal veins were the most numerous, longest, and most consistently spaced vessels of the transversely positioned veins (Fig. 5). The vessels were located in or near the hippocampal fissure and appeared to drain fields supplied by the internal transverse hippocampal arteries. The veins nearly paralleled the course of the accompanying arteries. At midpolar and temporal hippocampal regions, the vessels were oriented in the transverse plane in their caudorostral projection. For the hippocampus directly ventral to the corpus callosum, the vesscls followed a course nearly parallel to the sagittal plane.

An alternating pattern consisting of an internal transverse hippocampal artery and vein was readily observable in the double injected specimens (Fig. 6). The pattern was such that the arteries and veins alternated in sequence along the longitudinal hippocampal axis. Midpolar region measurements made of the dorsal-ventral distance between adjacent trunk vessels in coronal-sectioned specimens prepared for demonstration of only the arteries or the veins were about twice the value of those in doubleinjected or India ink-injected rats for demonstration of both arteries and veins. In nine hemispheres 109 measurements were made. The mean distance between the internal transverse hippocampal stem vessels was 230 $\mu \mathrm{m}$; standard deviation was $80 \mu \mathrm{m}$.

The external transverse hippocampal vcins wcrc most evident at midpolar and temporal regions of the hippocampus. The vessels followed a path approximately parallel to the corresponding arteries (Figs. 3, 5). Drainage of the extraventricular alveus, probably the adjacent stratum oriens, portions of cornu Ammonis within the area dentata, and the external blade of the fascia dentata was through these vessels which in turn drained into the longitudinal venous system. External transverse hippocampal veins draining the hippocampal formation beneath the corpus callosum also were observed to receive branches that penetrated and presumably drained ruclear masses of the thalamus. 


\section{DISCUSSION}

In rat, the longitudinal hippocampal artery was probably the homologue of the arcade vessel(s) observed by Nilges (12) for monkey, opossum, or the separate vessel illustrated for guinea pig. Anastomoses of the longitudinal artery with branches of the anterior cercbral artery or other named vessels deep in the transverse cerebral fissure were not ruled out due to the intermingling of vessels and difficulties encountered in tracing vessels as identified structures. Coyle (8) demonstrated anastomoses between branches of the longitudinal artery and the middle cerebral artery occur lateral to the rhinal fissure in neocortical regions. Supply to the hippocampal formation may occur through branches of the middle cerebral artery in event of a posterior cerebral artery occlusion.

Transverse vessels were located in the hippocampal fissure in all species observed by Nilges (12), Andersen et al. (2), and Coyle $(8,9)$. Clearly both arteries and veins were in the rat hippocampal fissure as detailed by the current study. The internal transverse vessels supplying and draining CA3 fields appeared to be paired and positioned at fairly consistent intervals. Such patterns were not evident elsewhere in the brain of rat and may reflect a basic lamellar neuronal organization of the hippocampus as suggested by Andersen et al. (2). Studies of the developing rat fascia dentata by Altman and Das (1), rabbit hippocampus by Stensaas (14, 15), and mouse hippocampus by Angevine (3) did not expose a segmental neuronal pattern along the longitudinal hippocampal axis during development. Pairing of artery and vein with supply and drainage to a particular tissue region may resemble the vascular pattern found by Wislocki and Campbell (16) and Scharrer (13) in the opossum brain. Hairpin capillary loops supplied exclusively by one artery would have to be demonstrated in the rat hippocampus before claims are made that hippocampal structures have a totally segmental vascular organization.

The spatial periodicity of the internal transverse hippocampal stem vessels seems to differ for different species. In mouse, Boast et al. (5) gave a spacing of about $150 \mu \mathrm{m}$, whereas in rat the distance between artery and vein was about $230 \mu \mathrm{m}$ as given here. The distance between arteries in rabbit was about $750 \mu \mathrm{m}$ according to Andersen et al. (2). Such differences may be real or may depend on the place of measurement, tissue shrinkage, nonfilling of some vessels, and other variables. Based on these figures, an electrode $500 \mu \mathrm{m}$ in diameter at its tip would either rupture or displace at least three transverse vessels in mouse, two in rat, or one in rabbit if the electrode were driven in the transverse plane through CA1 into. CA3 or the fascia dentata.

Consequences of an internal transverse hippocampal artery rupture 
could be grave. Although a relatively thin transverse slice of tissue may suffer an inadequate vascular supply, longitudinally directed association and commissural fiber bundles could be included in the lesion. Boast et al. $(4,5)$ provided evidence that electrode placements disrupting vessels in the hippocampal fissure were accompanied by measurable behavioral changes. Supply vessels to the rat fimbria, where longitudinally directed fibers are located, were very small and difficult to trace. Unpublished observations on several rabbits clearly revealed at least part of the arterial supply to the fimbria in that animal came from small branches of arteries coursing over and supplying the dorsal thalamus. Such vessels were not observed for rat but their existence was not ruled out.

The deep transverse hippocampal veins resembled the hippocampal subependymal veins described for human by Browning (6) and Wolf and Huang (17). According to Wolf and Huang (17) a separate longitudinal hippocampal vein anastomosed with the basal vein and drained all transverse vessels, a pattern not like those observed for rat. The hippocampal veins require additional comparative studies to clarify general spatial relations for a vertebrate series.

\section{REFERENCES}

1. Altman, J., and G. D. Das. 1965. Autoradiographic and histological evidence of postnatal hippocampal neurogenesis in rats. J. Comp. Neurol. 124 : 319-335.

2. Andersen, P., T. V. P. Bliss, and K. K. Skrede. 1971. Lamellar organization of hippocampal excitatory pathways. Exp. Brain Res. $13: 222-238$.

3. Angevine, J. B., JR. 1975. Development of the hippocampal region, pp. 61-94. In "The Hippocampus, Vol. 1: Structure and Development." R. L. Isaacson and K. H. Pribram [Eds.]. Plenum Press, New York.

4. Boast, C. A., Zornetzer, S. F., and M. E. Hamrick. 1975. Ferric ions located in hippocampal subfields of the mouse: Effects on behavior. T.I.T. J. Life Sci. 5: $11-16$.

5. Bonst, C. A., S. A. Reid, P. Johnson, and S. F. Zornetzer. 1976. A caution to brain scientists: Unsuspected hemorrhagic vascular damage resulting from mere electrode implantation. Brain Res. 103 : 527-534.

6. Browning, W. 1884. "The Veins of the Brain and Its Envclopes," pp. 1-79. F. B. O'Connor, Jr., Brooklyn, New York.

7. Coyle, P. 1975. Transverse and longitudinal hippocampal veins in rat. Anat. Rec. 181: $527-528$.

8. CoYle, P. 1975. Arterial patterns of the rat rhinencephalon and related structures. Exp. Neurol. 49 : 671-690.

9. Coyle, P. 1975. Vascular patterns in the rat hippocampus. Neurosci. Abstr. $1: 533$.

10 Lorente de Nó, R. 1933. Studies on the structure of the cerebral cortex. I. The area entorhinalis. J. Psychol. Neurol. 45 : 381-438.

11. LoRente de Nó, R. 1934. Studies on the structure of the cerebral cortex. II. Continuation of the study of the ammonic system. J. Psychol. Neurol. 46: 113-177.

12. Nilges, R. G. 1944. The arteries of the mammalian cornu ammonis. I. Comp. Neurol. 80: 177-190. 
13. Scharrer, E. 1940. Arteries and veins in the mammalian brain. Anat. Rec. 78 : 173-196.

14. Stensans, L. J. 1967. The development of hippocampal and dorsolateral pallial regions of the cerebral hemisphere in fetal rabbits. I. Fifteen millimeter stage, spongioblast morphology. J. Comp. Neurol. 129: 59-70.

15. StensaAs, L. J. 1967. The development of hippocampal and dorsolateral pallial regions of the cerebral hemisphere in fetal rabbits. II. Twenty millimeter stage, neuroblast morphology. J. Comp. Neurol. 129: 71-84.

16. Wislocki, G. B., and C. P. CAMPbell. 1936. The unusual manner of vascularization of the brain of the opossum. (Didelphys virginiana). Anat. Rec. 67: 177192.

17. Wolf, B. S., and Y. P. Huang. 1964. The subependymal veins of the lateral ventricles. Amer. J. Roentgenol. $91: 406-426$. 